\title{
Application of Federal Kalman Filter with Neural Networks in the Velocity and Attitude Matching of Transfer Alignment
}

\author{
Lijun Song $\mathbb{D}^{D}$, Zhongxing Duan $(\mathbb{D}$, Bo He, and Zhe Li \\ Electronic Information and Control Engineering College, Xi'an University of Architecture and Technology, Xi'an 710055, China \\ Correspondence should be addressed to Zhongxing Duan; zhx_duan@163.com
}

Received 8 July 2017; Accepted 8 October 2017; Published 21 January 2018

Academic Editor: Junpei Zhong

Copyright (C) 2018 Lijun Song et al. This is an open access article distributed under the Creative Commons Attribution License, which permits unrestricted use, distribution, and reproduction in any medium, provided the original work is properly cited.

\begin{abstract}
The centralized Kalman filter is always applied in the velocity and attitude matching of Transfer Alignment (TA). But the centralized Kalman has many disadvantages, such as large amount of calculation, poor real-time performance, and low reliability. In the paper, the federal Kalman filter (FKF) based on neural networks is used in the velocity and attitude matching of TA, the Kalman filter is adjusted by the neural networks in the two subfilters, the federal filter is used to fuse the information of the two subfilters, and the global suboptimal state estimation is obtained. The result of simulation shows that the federal Kalman filter based on neural networks is better in estimating the initial attitude misalignment angle of inertial navigation system (INS) when the system dynamic model and noise statistics characteristics of inertial navigation system are unclear, and the estimation error is smaller and the accuracy is higher.
\end{abstract}

\section{Introduction}

Because there are too many vector dimensions in TA, the centralized Kalman filter has many disadvantages, such as large amount of calculation, poor real-time performance, and low reliability. In theory, the federal filtering is optimal or suboptimal, and it has the characteristics of high reliability, design flexibility, and being easy to apply to data fusion of navigation. In the engineering practice, system noise and measurement noise are always random signal, so it is difficult to get the statistic characteristics. The federal Kalman filter with neural networks is proposed in the paper. The federal Kalman filter is combined with the neural networks to improve the precision of initial attitude misalignment angle of inertial navigation system $[1,2]$.

\section{The Theory of Federal Kalman Filter with Neural Networks}

2.1. The Traditional Kalman Filter. It is assumed that the system equation and measurement equation of linear discrete system can be written as follows:

$$
\begin{aligned}
\mathbf{X}_{k} & =\boldsymbol{\Phi}_{k, k-1} \mathbf{X}_{k-1}+\mathbf{W}_{k-1} \\
\mathbf{Z}_{k} & =\mathbf{H}_{k} \mathbf{X}_{k}+\mathbf{V}_{k},
\end{aligned}
$$

where $\mathbf{X}_{k}$ is estimated state, $\mathbf{Z}_{k}$ is the measurement of system, and $\boldsymbol{\Phi}_{k, k-1}$ is transfer matrix from $t_{k-1}$ to $t_{k} \cdot \mathbf{W}_{k-1}$ is noise sequence of system incentive. $\mathbf{H}_{k}$ is the measurement matrix, $\mathbf{V}_{k}$ is noise sequence of measurement, and $E\left[\mathbf{V}_{k} \mathbf{V}_{k}^{T}\right]=\mathbf{R}$.

So the Kalman filter equation is shown as follows.

(1) Time update is

$$
\begin{aligned}
\widehat{\mathbf{X}}_{k / k-1} & =\boldsymbol{\Phi}_{k, k-1} \widehat{\mathbf{X}}_{k-1} \\
\mathbf{P}_{k / k-1} & =\boldsymbol{\Phi}_{k, k-1} \mathbf{P}_{k-1} \boldsymbol{\Phi}_{k, k-1}^{T}+\boldsymbol{\Gamma}_{k-1} \mathbf{Q}_{k-1} \boldsymbol{\Gamma}_{k-1}^{T} .
\end{aligned}
$$

(2) Measurement update is

$$
\begin{aligned}
& \mathbf{K}_{k}=\mathbf{P}_{k / k-1} \mathbf{H}_{k}^{T}\left(\mathbf{H}_{k} \mathbf{P}_{k / k-1} \mathbf{H}_{k}^{T}+\mathbf{R}_{k}\right)^{-1} \\
& \widehat{\mathbf{X}}_{k}=\widehat{\mathbf{X}}_{k / k-1}+\mathbf{K}_{k}\left(\mathbf{Z}_{k}-\mathbf{H}_{k} \widehat{\mathbf{X}}_{k / k-1}\right) \\
& \mathbf{P}_{k}=\left(\mathbf{I}-\mathbf{K}_{k} \mathbf{H}_{k}\right) \mathbf{P}_{k / k-1}\left(\mathbf{I}-\mathbf{K}_{k} \mathbf{H}_{k}\right)^{T}+\mathbf{K}_{k} \mathbf{R}_{k} \mathbf{K}_{k}^{T} .
\end{aligned}
$$

The state estimate $\mathbf{X}_{k}$ could be calculated when the initial values $\mathbf{X}_{0}$ and $\mathbf{P}_{0}$ are known.

2.2. The Federal Filter. The federal filter is composed of several subfilters and a main filter, where the subfilter can 


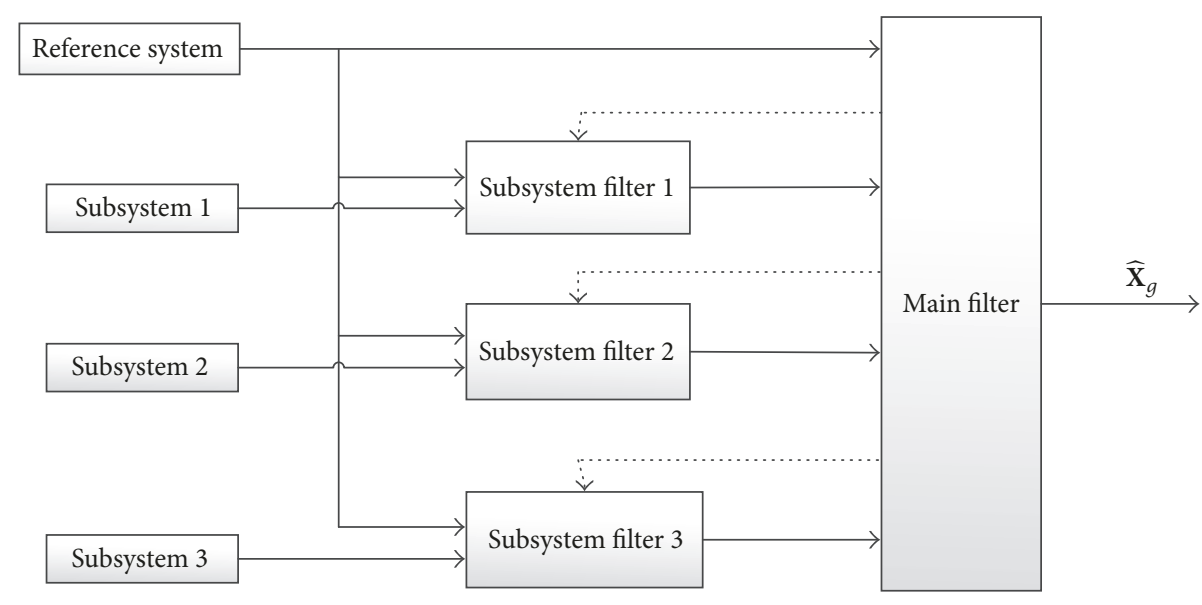

FIgURE 1: The structure of the federal filter.

independently update the time and measurement, and the result will be sent to the main filter. And the main filter will feed back to the subfilter after data fusion is analyzed. It is the initial value of the next cycle. The federal filter is that the large matrix of centralized filter algorithm is divided into the subfilters $[3,4]$. The structure of the federal filter is shown in Figure 1.

The fusion algorithm of the traditional federal filter is as follows:

$$
\begin{aligned}
\widehat{\mathbf{X}}_{g} & =\mathbf{P}_{g}\left(\sum_{i=1}^{m} \mathbf{P}_{c i}^{-1} \widehat{\mathbf{X}}_{c i}\right) \\
\mathbf{P}_{g} & =\left(\sum_{i=1}^{m} \mathbf{P}_{c i}^{-1}\right)^{-1},
\end{aligned}
$$

where $\widehat{\mathbf{X}}_{g}$ is global state estimation after fusion, $\widehat{\mathbf{X}}_{c i}$ is the local optimum estimation of subfilter on the system of public state, $\mathbf{P}_{c i}$ is variance matrix of error of the local optimum estimation, and the local estimations are not related to each other.

2.3. The BP Neural Networks. The uptime of Kalman filter is proportional to the cubic of the system order. The Kalman filter will lose the real-time performance when the order of system is very high, so the precision is not improved by the order of system.

Artificial neural network is a system that simulates the structure and function of human brain neuron networks by method of engineering technology. It is comprised of a large number of simple nonlinear processing units, and there are complex and flexible connections between units.

The neural networks have a strong self-learning ability and arbitrary nonlinear functions and parallel processing could be realized. So the neural networks are always applied in the information technology and control engineering. According to the characteristics of information transmission, neural networks can be divided into feedforward and feedback. The BP neural networks are feedforward networks which are based on error backpropagation algorithm [5-7].

There are input layer nodes, one or more hidden-layer nodes, and output layer nodes in the BP neural networks.
If the number of hidden-layers and nodes in each layer are determined, the structure of the BP neural networks is determined [8-10]. The principle of BP neural networks is shown as Figure 2.

\subsection{The Federal Kalman Filter with Neural Networks}

2.4.1. The Theory of Federal Kalman Filter. The theory of federal Kalman filters in TA is as follows:

(1) The output date of master inertial navigation system (MINS) is used as the common reference system, the velocity and angularity of slaver inertial navigation system (SINS) regarded as independent subsystems. In order to solve the problem, the BP neural networks are used in the two subsystems while the statistics characteristics of the system dynamic model and noise model are unclear.

(2) The output date of two subsystems used the federal filter to obtain global suboptimal estimation.

2.4.2. The Theory of Kalman Filter with Neural Networks. The purpose of the subfilter which used the BP neural networks is that the Kalman filter is followed by the BP neural networks with the minimum error.

The training process of neural networks is as follows: first, the Kalman filter worked as open loop state to eliminate the influence of convergence rate for initial value. Second, the Kalman filter worked as closed loop state, and the initial sample values of the neural network are constituted by the input and output of the Kalman filter. Lastly, the sample value of training could be got by averaging the testing values of measurements. After the training, The BP neural networks will be worked alone when the Kalman filter is removed [1113].

The inputs of BP neural networks are comprised of three parts: (1) the difference of state prediction and state estimation is $\widehat{\mathbf{X}}_{k}-\widehat{\mathbf{X}}_{k / k-1}$. (2) The difference of observation and observation estimation is $\mathbf{Z}_{k}-\mathbf{H}_{k} \widehat{\mathbf{X}}_{k / k-1}$. (3) The filter gain is $\mathbf{K}_{k} \cdot \Delta \widehat{\mathbf{X}}_{k}=D-\widehat{\mathbf{X}}_{k}$ is treated as the desired output of BP neural networks, where $D$ is the theoretical value of state vector. $\Delta \widehat{\mathbf{X}}_{k}$ 


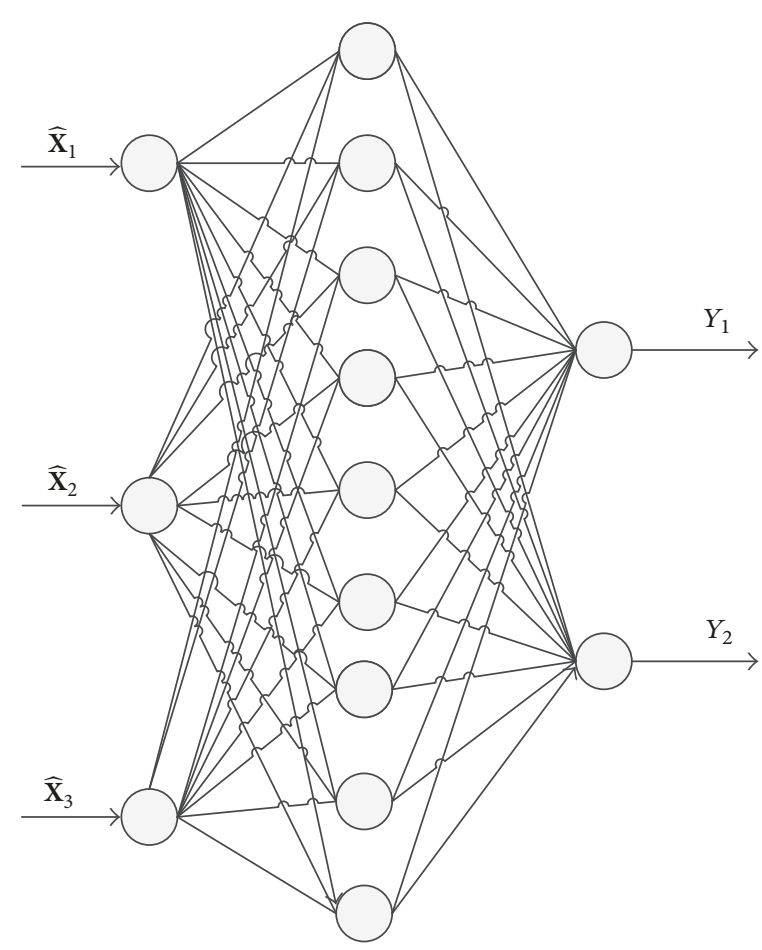

FIGURE 2: The principle of BP neural networks.

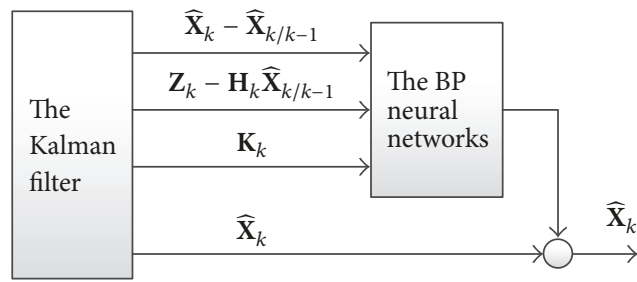

FIGURE 3: The principle of Kalman filters with neural networks.

is the output of BP neural networks and $\widehat{\mathbf{X}}_{k}$ is the output of Kalman filter, where the two outputs are added together after the BP neural networks are trained by samples.

The principle of Kalman filters with neural networks is shown as Figure 3.

\section{The Velocity and Attitude Matching in TA}

The velocity matching in TA is required to assist maneuver (such as right circle), and attitude matching in TA is required angular velocity of pitch or roll (such as wing maneuver), but it is not separated from the head platform misalignment angle in the velocity matching and the same in the attitude matching when the plane has the wing maneuver $[13,14]$.

In the velocity and attitude matching, horizontal alignment is realized in the velocity and azimuth alignment is realized in attitude matching. The wing maneuver is required during the alignment procedure.

\subsection{State Equation of the Velocity and Attitude Matching. It}

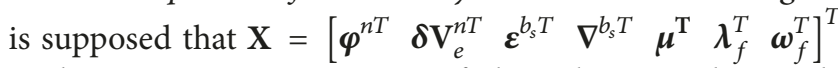
is the system state equation of the velocity and attitude matching, where $\varphi^{n}=\left[\begin{array}{lll}\varphi_{x} & \varphi_{y} & \varphi_{z}\end{array}\right]^{T}$ is the platform misalignment angle of slaver inertial navigation system, $\boldsymbol{\delta} \mathbf{V}_{e}^{n}=\left[\begin{array}{lll}\delta \mathrm{V}_{e x}^{n} & \delta \mathrm{V}_{e y}^{n} & \delta \mathrm{V}_{e z}^{n}\end{array}\right]^{T}$ is velocity error of slaver inertial navigation system, $\boldsymbol{\mu}=\left[\begin{array}{lll}\mu_{x} & \mu_{y} & \mu_{z}\end{array}\right]^{T}$ is error of missile body's installation angle, $\nabla^{b_{s}}=\left[\begin{array}{ccc}\nabla_{x}^{b_{s}} & \nabla_{y}^{b_{s}} & \nabla_{z}^{b_{s}}\end{array}\right]^{T}$ is accelerometer's constant error of slaver inertial navigation system, $\lambda_{\mathrm{f}}=$ $\left[\begin{array}{lll}\lambda_{f x} & \lambda_{f y} & \lambda_{f z}\end{array}\right]^{T}$ is flexure deformation angle of wing, and $\boldsymbol{\omega}_{\mathbf{f}}=\left[\begin{array}{lll}\omega_{f x} & \omega_{f y} & \omega_{f z}\end{array}\right]^{T}$ is flexure deformation angular rate of wing. State equation of the velocity and attitude matching is

$$
\begin{aligned}
\dot{\boldsymbol{\varphi}}^{n}= & -\boldsymbol{\omega}_{i n}^{n} \times \boldsymbol{\varphi}^{n}-\mathbf{C}_{b_{s}}^{n} \boldsymbol{\varepsilon}_{b}^{b_{s}}-\mathbf{C}_{b_{s}}^{n} \boldsymbol{\varepsilon}_{w}^{b_{s}} \\
\delta \dot{\mathbf{V}}^{n}= & \left(\mathbf{C}_{b_{s}}^{n} \mathbf{f}^{b_{s}}\right) \times \boldsymbol{\varphi}-\left(2 \boldsymbol{\omega}_{i e}^{n}+\boldsymbol{\omega}_{e n}^{n}\right) \times \delta \mathbf{V}^{n}+\mathbf{C}_{b_{s}}^{n} \nabla_{b}^{b_{s}} \\
& +\mathbf{C}_{b_{s}}^{n} \nabla_{w}^{b_{s}} \\
\dot{\boldsymbol{\varepsilon}}^{b_{s}}= & \mathbf{0} \\
\dot{\nabla}^{b_{s}}= & \mathbf{0} \\
\dot{\boldsymbol{\mu}}^{b_{f}}= & \mathbf{0}
\end{aligned}
$$




$$
\begin{aligned}
\dot{\boldsymbol{\lambda}}_{f} & =\boldsymbol{\omega}_{f} . \\
\dot{\boldsymbol{\omega}}_{f} & =-\left[\boldsymbol{\beta}^{2}\right] \boldsymbol{\lambda}_{f}-[\boldsymbol{\beta}] \boldsymbol{\omega}_{f}+\boldsymbol{\eta}
\end{aligned}
$$

So the state-space model of the velocity and attitude matching is

(5)

$$
\dot{\mathbf{X}}=\left[\begin{array}{ccccccc}
-\left(\boldsymbol{\omega}_{i n}^{n} \times\right) & \mathbf{0}_{3 \times 3} & -\mathbf{C}_{b_{s}}^{n} & \mathbf{0}_{3 \times 3} & \mathbf{0}_{3 \times 3} & \mathbf{0}_{3 \times 3} & \mathbf{0}_{3 \times 3} \\
\left(\mathbf{C}_{b_{s}}^{n} \mathbf{f}^{b_{s}} \times\right) & -\left(\left(2 \boldsymbol{\omega}_{i e}^{n}+\boldsymbol{\omega}_{e n}^{n}\right) \times\right) & \mathbf{0}_{3 \times 3} & \mathbf{C}_{b_{s}}^{n} & \mathbf{0}_{3 \times 3} & \mathbf{0}_{3 \times 3} & \mathbf{0}_{3 \times 3} \\
\mathbf{0}_{3 \times 3} & \mathbf{0}_{3 \times 3} & \mathbf{0}_{3 \times 3} & \mathbf{0}_{3 \times 3} & \mathbf{0}_{3 \times 3} & \mathbf{0}_{3 \times 3} & \mathbf{0}_{3 \times 3} \\
\mathbf{0}_{3 \times 3} & \mathbf{0}_{3 \times 3} & \mathbf{0}_{3 \times 3} & \mathbf{0}_{3 \times 3} & \mathbf{0}_{3 \times 3} & \mathbf{0}_{3 \times 3} & \mathbf{0}_{3 \times 3} \\
\mathbf{0}_{3 \times 3} & \mathbf{0}_{3 \times 3} & \mathbf{0}_{3 \times 3} & \mathbf{0}_{3 \times 3} & \mathbf{0}_{3 \times 3} & \mathbf{0}_{3 \times 3} & \mathbf{0}_{3 \times 3} \\
\mathbf{0}_{3 \times 3} & \mathbf{0}_{3 \times 3} & \mathbf{0}_{3 \times 3} & \mathbf{0}_{3 \times 3} & \mathbf{0}_{3 \times 3} & \mathbf{0}_{3 \times 3} & I_{3 \times 3} \\
\mathbf{0}_{3 \times 3} & \mathbf{0}_{3 \times 3} & \mathbf{0}_{3 \times 3} & \mathbf{0}_{3 \times 3} & \mathbf{0}_{3 \times 3} & -\left[\boldsymbol{\beta}^{2}\right] & -[\boldsymbol{\beta}]
\end{array}\right] \mathbf{X}+\left[\begin{array}{c}
-\mathbf{C}_{b_{s}}^{n} \boldsymbol{\varepsilon}_{w}^{b_{s}} \\
\mathbf{C}_{b_{s}}^{n} \nabla_{w}^{b_{s}} \\
\mathbf{0}_{3 \times 3} \\
\mathbf{0}_{3 \times 3} \\
\mathbf{0}_{3 \times 3} \\
\mathbf{0}_{3 \times 3} \\
\eta
\end{array}\right]
$$

where $\mathbf{C}_{b_{s}}^{n}=\left[\begin{array}{lll}T_{11} & T_{12} & T_{13} \\ T_{22} & T_{22} \\ T_{31} & T_{32} & T_{33}\end{array}\right]$ is the attitude matrix of slaver inertial navigation system:

$$
\begin{aligned}
\left(\mathbf{C}_{b_{s}}^{n} \mathbf{b}^{b_{s}} \times\right) & =\left[\begin{array}{ccc}
0 & -f_{U}^{n} & f_{N}^{n} \\
f_{U}^{n} & 0 & -f_{E}^{n} \\
-f_{N}^{n} & f_{E}^{n} & 0
\end{array}\right] \\
\left(\left(2 \boldsymbol{\omega}_{i e}^{n}+\boldsymbol{\omega}_{e n}^{n}\right) \times\right) & =\left[\begin{array}{ccc}
\left(2 \omega_{i e} \sin L+\frac{V_{E} \tan L}{R_{N}}\right) & -\left(2 \omega_{i e} \sin L+\frac{V_{E} \tan L}{R_{N}}\right) & \left(2 \omega_{i e} \cos L+\frac{V_{E}}{R_{N}}\right) \\
-\left(2 \omega_{i e} \cos L+\frac{V_{E}}{R_{N}}\right) & 0 & \frac{V_{N}}{R_{M}} \\
R_{M} & 0
\end{array}\right],
\end{aligned}
$$

where $\boldsymbol{\varepsilon}_{w}^{b_{s}}$ is Gaussian white noise of gyro, $\nabla_{w}^{b_{s}}$ is Gaussian white noise of accelerometer, $\boldsymbol{\eta}=\left[\begin{array}{lll}\eta_{x} & \eta_{y} & \eta_{z}\end{array}\right]^{T}$ is Gaussian white noise sequence of second order, in which $\eta_{i} \sim \mathbf{N}\left(0, Q_{i}\right)$, $Q_{i}=4 \beta_{i}^{3} \sigma_{\eta}^{2}$, and $\sigma_{\eta}^{2}$ is the variance of flexure deformation angle; $[\boldsymbol{\beta}]=\operatorname{diag}\left(\beta_{x}, \beta_{y}, \beta_{z}\right)$ and $\left[\boldsymbol{\beta}^{2}\right]=\operatorname{diag}\left(\beta_{x}^{2}, \beta_{y}^{2}, \beta_{z}^{2}\right)$.

3.2. The Measurement Equation of Velocity and Attitude Matching. It is supposed that $\widehat{\mathbf{V}}_{e m}^{n}$ is the velocity of MINS, $\widehat{\mathbf{V}}_{e s}^{n}$ is the velocity of SINS, $\widehat{\mathbf{V}}_{L A}^{n}$ is the lever velocity from MINS, $\widehat{\mathbf{C}}_{b_{m}}^{n}$ is the attitude matrix of MINS, $\widehat{\mathbf{C}}_{b_{s}}^{n}$ is the attitude matrix of SINS, and $\mathbf{C}_{b_{f}}^{b_{h}}$ is transformation matrix between $b_{f}$ and $b_{h}$, where $b_{f}$ is installation coordinate and $b_{h}$ is horizontal coordinate.

The difference of velocity between MINS and SINS is the measurement in the velocity matching; attitude matrix is the measurement in the attitude matching. The measurement of the velocity and attitude matching is as follows.

Consider

$$
\mathbf{Z}_{V}=\widehat{\mathbf{V}}_{e s}^{n}-\left(\widehat{\mathbf{V}}_{e m}^{n}+\widehat{\mathbf{V}}_{L A}^{n}\right)
$$

$$
\begin{aligned}
\mathbf{Z} & =\left[\begin{array}{l}
Z_{V} \\
Z_{\theta}
\end{array}\right] \\
\mathbf{Z}_{\theta} & =\left[\begin{array}{l}
\frac{\mathbf{Z}_{\mathrm{DCM}}(3,2)-\mathbf{Z}_{\mathrm{DCM}}(2,3)}{2} \\
\frac{\mathbf{Z}_{\mathrm{DCM}}(1,3)-\mathbf{Z}_{\mathrm{DCM}}(3,1)}{2} \\
\frac{\mathbf{Z}_{\mathrm{DCM}}(2,1)-\mathbf{Z}_{\mathrm{DCM}}(1,2)}{2}
\end{array}\right] \\
\mathbf{Z}_{\mathrm{DCM}} & =\widehat{\mathbf{C}}_{b_{m}}^{n} \mathbf{C}_{b_{f}}^{b_{h}} \widehat{\mathbf{C}}_{n}^{b_{s}} \\
& =\left[\mathbf{I}-\left(\boldsymbol{\varphi}_{m}^{n} \times\right)\right] \mathbf{C}_{b_{m}}^{n} \mathbf{C}_{b_{f}}^{b_{h}} \mathbf{C}_{n}^{b_{s}}\left[\mathbf{I}+\left(\boldsymbol{\varphi}^{n} \times\right)\right] .
\end{aligned}
$$

$\boldsymbol{\varphi}_{m}^{n}$ is the attitude error angle of master inertial navigation system, and $\varphi^{n}$ is the attitude error angle of slaver inertial navigation system.

The measurement equation of velocity and attitude matching is 


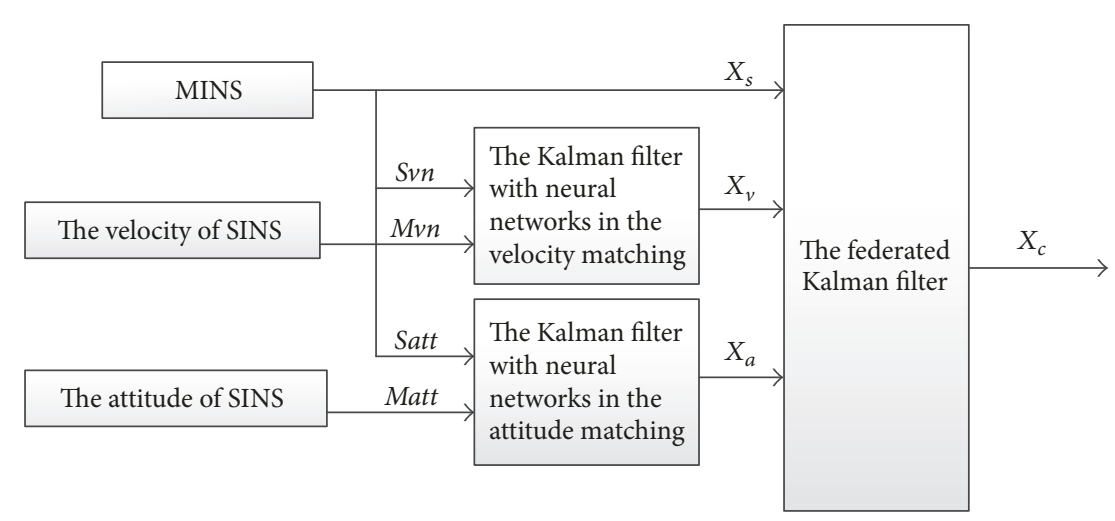

FIgURE 4: The principle diagram of the federated Kalman filter with neural networks.

$$
\begin{aligned}
\mathbf{Z}= & {\left[\begin{array}{ccccccc}
\mathbf{0}_{3 \times 3} & \mathbf{I}_{3 \times 3} & \mathbf{0}_{3 \times 3} & \mathbf{0}_{3 \times 3} & \mathbf{0}_{3 \times 3} & \mathbf{0}_{3 \times 3} & \mathbf{0}_{3 \times 3} \\
\mathbf{I}_{3 \times 3} & \mathbf{0}_{3 \times 3} & \mathbf{0}_{3 \times 3} & \mathbf{0}_{3 \times 3} & -\mathbf{C}_{b_{m}}^{n} \mathbf{C}_{b_{f}}^{b_{h}} & -\mathbf{C}_{b_{m}}^{n} & \mathbf{0}_{3 \times 3}
\end{array}\right] \mathbf{X} } \\
& +\left[\begin{array}{c}
\mathbf{V}_{V} \\
\mathbf{V}_{\boldsymbol{\theta}}
\end{array}\right]
\end{aligned}
$$

where the white noise of zero mean Gaussian is $\mathbf{V}_{V}$, and the measurement noise signal of unknown is $\mathbf{V}_{\theta}$.

\section{Application of Federal Kalman Filter with Neural Networks in the Velocity and Attitude Matching}

4.1. The Structure Design of Federal Kalman Filter with Neural Networks. The centralized Kalman filter is applied in the velocity and attitude matching of TA. It is superior to the velocity matching or the attitude matching, especially in the speed of alignment and the requirement of maneuver, but there are too many numbers of dimensions and large amount of calculation. Combined with the neural networks, the performance of federal Kalman filter is improved on the velocity and attitude matching when the statistics characteristics of the system dynamic model and noise model are unclear [15, 16 .

The parallel computation is supported by the federal Kalman filter with neural networks to reduce the calculation in TA, and the neural networks are used in the subfilter. The principle diagram of the federal Kalman filter with neural networks on the velocity and attitude matching is shown as Figure 4.

4.2. The Simulation of Federal Kalman Filter with Neural Networks in the Velocity and Attitude Matching. It is supposed that there are the flexure deformation of the wing and the unknown measurement noise signal when the federal Kalman filters with neural networks are simulated in the velocity and attitude matching.

According to the fight characteristic, this paper makes the corresponding numerical simulation under wing motion by aircraft. The time of shake wing is $60 \mathrm{~s}$ and the angle of shake wing is $30^{\circ}$. The initial position of TA is that north latitude is $34.03006^{\circ}$, east longitude is $108.76405^{\circ}$, and altitude is $448 \mathrm{~m}$; the initial attitude of TA is that yaw is $-90^{\circ}$, roll is $0^{\circ}$, and pitch is $0^{\circ}$.

The condition for simulation is as follows: error parameters of SINS: constant drift of gyro is $1^{\circ} / \mathrm{h}$, random walk of gyro is $0.1^{\circ} / \sqrt{\mathrm{h}}$, constant offset error of accelerometer is $5 \times$ $10^{-4} \mathrm{~g}$, and standard deviation of accelerometer is $5 \times 10^{-5} \mathrm{~g}$. $\sqrt{\mathrm{s}}$.

$$
\begin{aligned}
& \text { Installing-error angle of missile body: } \boldsymbol{\mu}= \\
& {\left[\begin{array}{lll}
0.1^{\circ} & 0.1^{\circ} & 0.1^{\circ}
\end{array}\right]^{T} \text {. }} \\
& \text { Misalignment initial angle of SINS: } \boldsymbol{\varphi}(0)= \\
& {\left[\begin{array}{lll}
0.1^{\circ} & 0.1^{\circ} & 0.5^{\circ}
\end{array}\right]^{T} \text {. }} \\
& \text { Velocity initial error of SINS: } \delta \mathbf{V}_{e}^{n}(0)= \\
& {\left[\begin{array}{lll}
3 \mathrm{~m} / \mathrm{s} & 3 \mathrm{~m} / \mathrm{s} & 3 \mathrm{~m} / \mathrm{s}
\end{array}\right]^{T} .}
\end{aligned}
$$

The number of input layer nodes of BP neural networks is 6 , the number of hidden-layer nodes of BP neural networks is 12 , and the number of output layer nodes of BP neural networks is 3 .

The simulation period is $20 \mathrm{~ms}$. Meanwhile, it has the same conditions as Kalman filter. The blue solid line is the result of the federal Kalman filter with neural networks, and the red dashed line is the result of Kalman filter.

It is the main difference between the federal Kalman filter with neural networks and the Kalman filter that the Kalman filters with neural networks are used in the velocity matching and the attitude matching, and the result is got by the Kalman filter with neural networks in the velocity matching and the attitude matching. In order to get the optimal estimation of navigation parameter error, the result is sent to the main filter for data fusion.

From Figure 5, it is showed that the convergence of misalignment angle of the Kalman filter has been in $10^{\prime}$ after $20 \mathrm{~s}$. But the convergence of misalignment angle of the federal Kalman filter with neural networks has been in $5^{\prime}$ after $20 \mathrm{~s}$. The precision of federal Kalman filter with neural networks is above the precision of Kalman filter. 


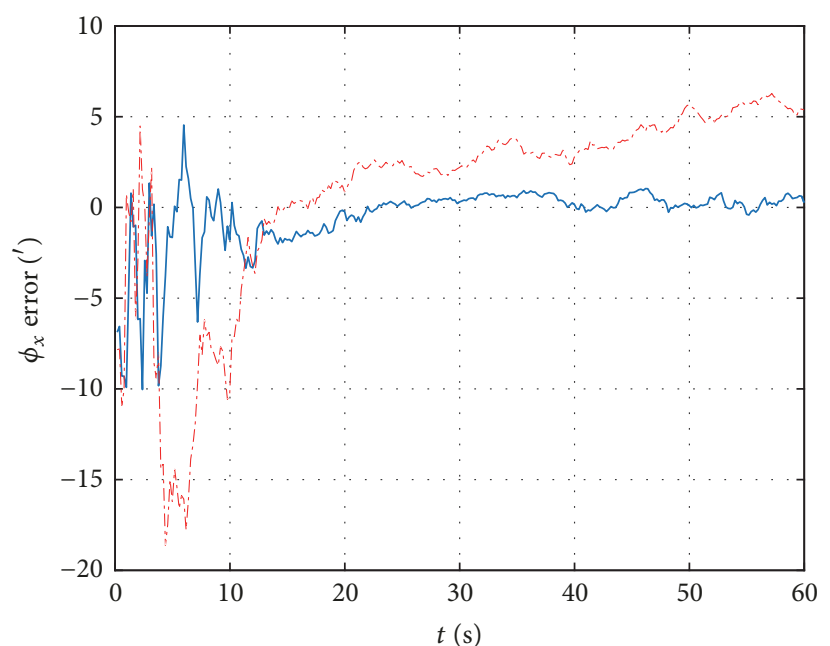

FKF with neural networks $\mathrm{CKF}$

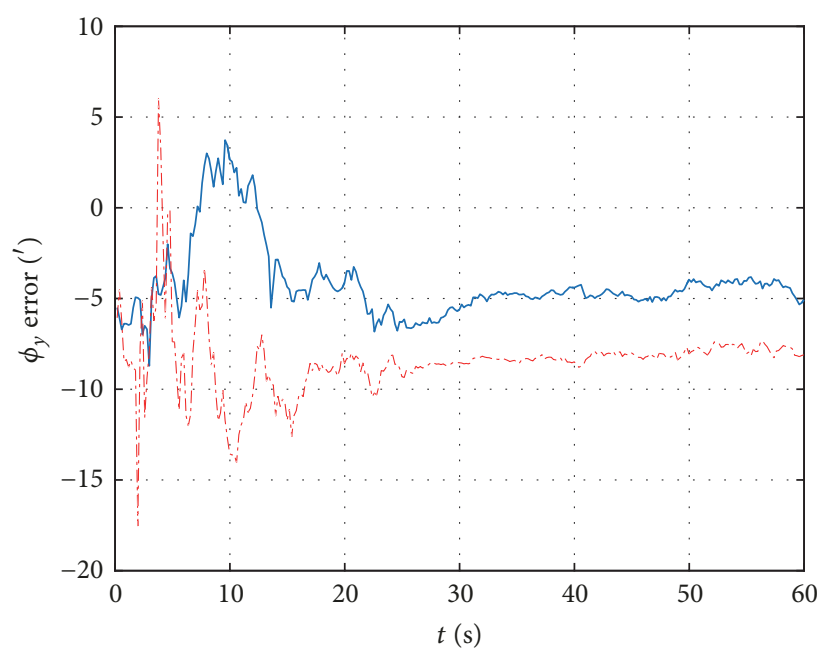

FKF with neural networks

-.. $\mathrm{CKF}$

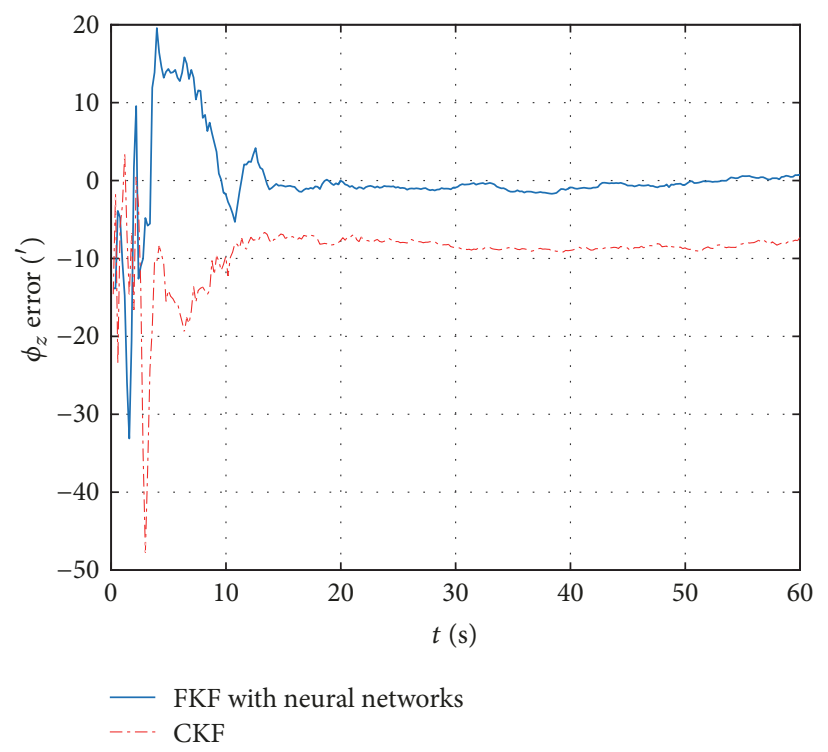

FIgURE 5: Estimate error of misalignment angle.

\section{Summary}

The input of the networks is the error parameters of Kalman filter. After the sample training, the BP networks output is added to the results of Kalman filter. In the practical engineering applications, this algorithm is superior to the Kalman filter under the nonlinear situation, and the result of simulation shows that the federal Kalman filter with neural networks in the velocity and attitude matching is more practicable and effective. The federal Kalman filter with neural networks is better to estimate the initial attitude misalignment angle of inertial navigation system when the system dynamic model and noise statistics characteristics of inertial navigation system are unclear, and the estimation error is smaller and the accuracy is higher.

\section{Conflicts of Interest}

The authors declare that they do not have any commercial or associative interest that represents conflicts of interest in connection with the work submitted.

\section{Acknowledgments}

This work is partially supported by the special scientific research project of the Education Department of Shaanxi Provincial Government, School Foundation Research Fund, Natural Science Foundation of Shaanxi Provincial Department and the National Natural Science Foundation of China, the project Number: 15JK1416, JC1702, 2016JM6084 and 51678470 . 


\section{References}

[1] X.-J. Guan and X.-L. Wang, "Transfer alignment match methods for strapdown inertial navigation system on moving bases," Aero Weapons, vol. 4, no. 2, pp. 3-8, 2014.

[2] G.-l. Yang, L.-f. Wang, E.-k. Yuan, L. Cai, and L.-w. Qiao, "Rapid transfer alignment for carrier - based aircrafts in catapult," in Ship Science and Technolog, vol. 36, pp. 50-54, 3 edition, 2014.

[3] L. Zhang, S. Qian, S. Zhang, and H. Cai, "Federated nonlinear predictive filtering for the gyroless attitude determination system," Advances in Space Research, vol. 58, no. 9, pp. 1671-1681, 2016.

[4] Q. Y. yuan and N. I. Huifang, "Application of federated filtering theory to designing integrated navigation system," Journal of Chinese Inertial Technology, vol. 5, no. 3, pp. 1-5, 1997.

[5] C. Yang, Y. Jiang, Z. Li, W. He, and C.-Y. Su, "Neural control of bimanual robots with guaranteed global stability and motion precision," IEEE Transactions on Industrial Informatics, 2017.

[6] L. Dan, "Improved BP neural network WSN data fusion scheme," Information Technology, vol. 37, no. 2, pp. 155-160, 2017.

[7] C. Yang, J. Luo, Y. Pan, Z. Liu, and C. Y. Su, "Personalized variable gain control with tremor attenuation for robot teleoperation," IEEE Transactions on Systems, Man, and Cybernetics: Systems, pp. 1-12, 2017.

[8] M. Yongjun, X. Yonghao, L. Yang, and L. Yajun, "Data aggregation algorithm based on the model of deep learning," Journal of Tianjin University of Science Technology, vol. 32, no. 4, pp. 1-5, 2017.

[9] C. Yang, K. Huang, H. Cheng, Y. Li, and C.-Y. Su, "Haptic identification by ELM-controlled uncertain manipulator," IEEE Transactions on Systems, Man, and Cybernetics: Systems, vol. 47, no. 8, pp. 2398-2409, 2017.

[10] T. Zengshan and C. Yongquan, "Attitude measurement fusion algorithm in GPS/SINS based on BP neural-network," Journal of Chongqing University of Posts and Telecommunications, vol. 26, no. 4, pp. 478-482, 2014.

[11] X. Tianlai and M. Xu, "INS/GPS integrated navigation method based on elman neural network," in Proceedings of the International Conference on Fuzzy Systems and Neural Computing, pp. 310-313, 2011.

[12] B. Feng, H. Ma, M. Fu, and C. Yang, "Real-time state estimator without noise covariance matrices knowledge-fast minimum norm filtering algorithm," IET Control Theory \& Applications, vol. 9, no. 9, pp. 1422-1432, 2015.

[13] G.-M. Yan, J. Weng, P.-X. Yang, and Y.-y. Qin, "Study on SINS rapid gyrocompass initial alignment," International Symposium on Inertial Technology and Navigation, pp. 323-330, 2010.

[14] K. Kim, S. Seol, and S.-H. Kong, "High-speed train navigation system based on multi-sensor data fusion and map matching algorithm," International Journal of Control, Automation, and Systems, vol. 13, no. 3, pp. 503-512, 2015.

[15] C. Yang, X. Wang, Z. Li, Y. Li, and C. Su, "Teleoperation control based on combination of wave variable and neural networks," IEEE Transactions on Systems, Man, and Cybernetics: Systems, vol. 47, no. 8, pp. 2125-2136, 2017.

[16] L. Bin, The research and design of transfer alignment simulation and verification system on ship carried weapon inertial navigation system, Harbin Engineering University, Harbin, China, 2012. 


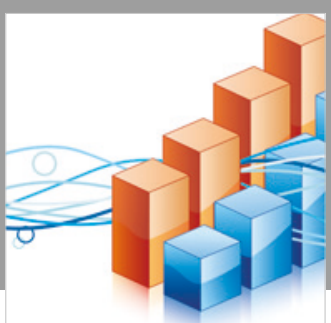

Advances in

Operations Research

\section{-n-m}
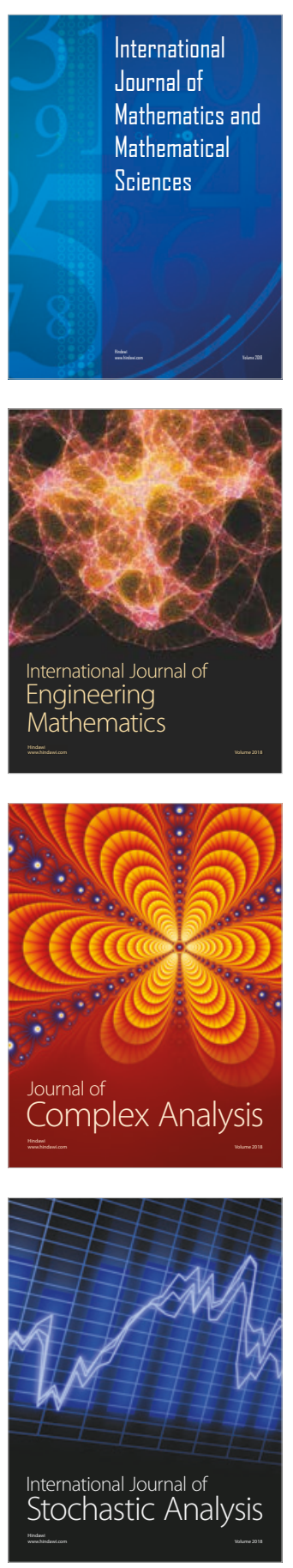
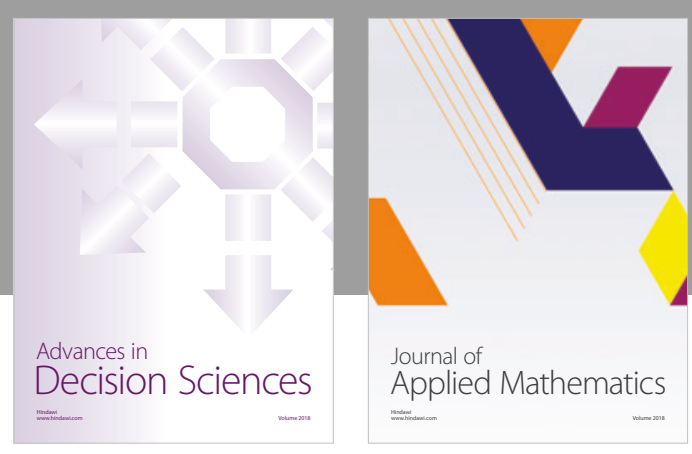

Journal of

Applied Mathematics
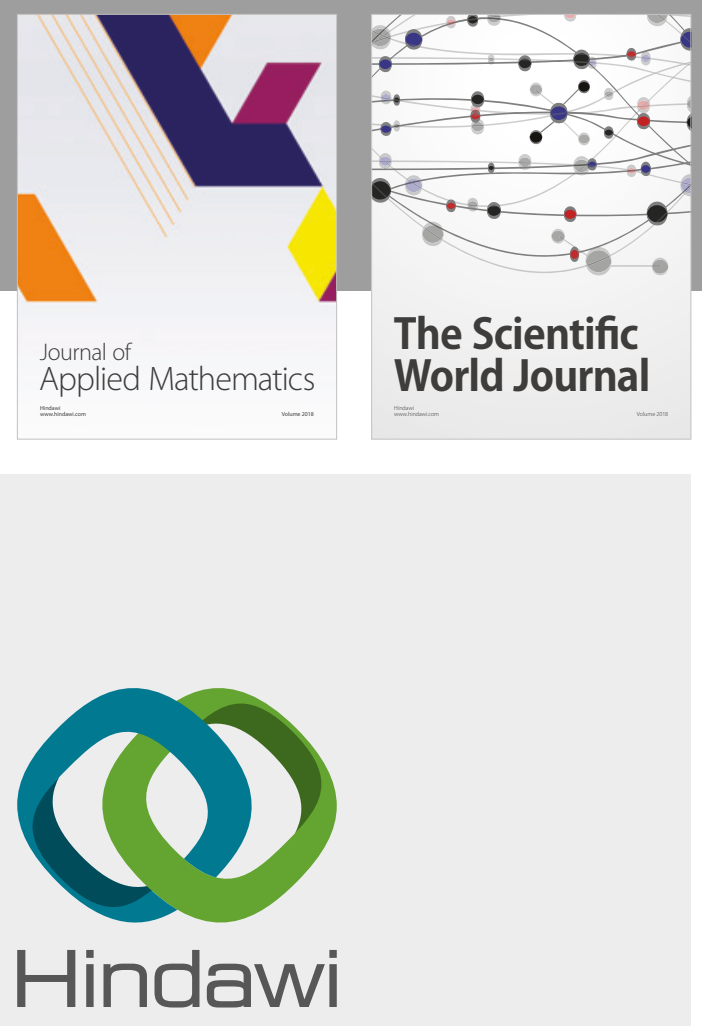

Submit your manuscripts at

www.hindawi.com

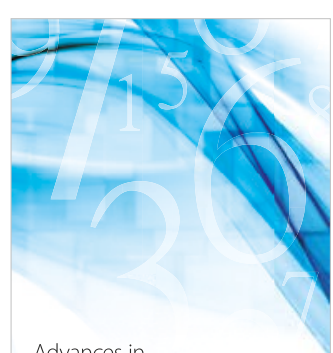

Advances in
Numerical Analysis
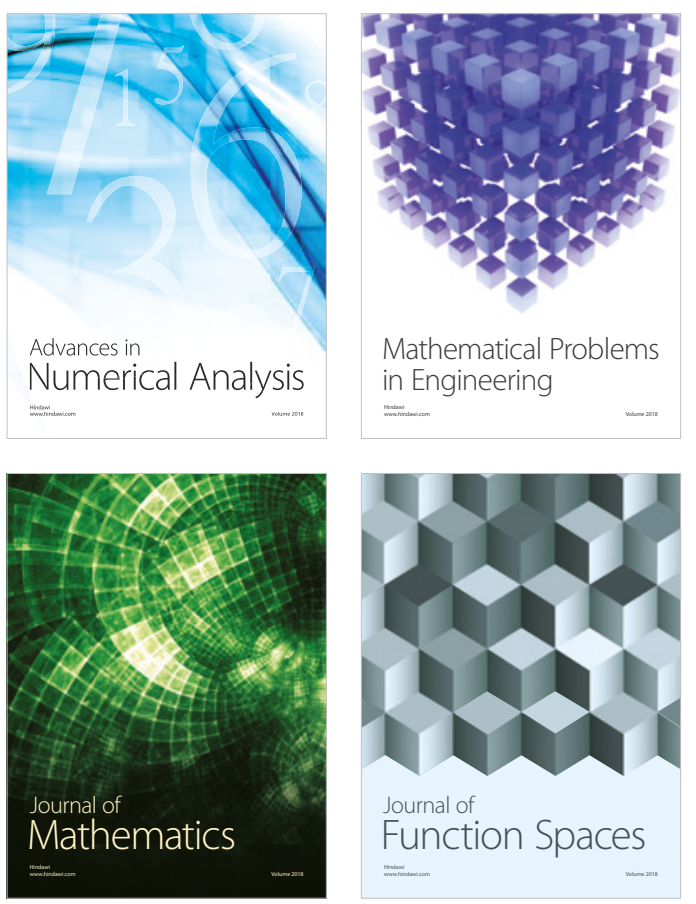

Mathematical Problems in Engineering

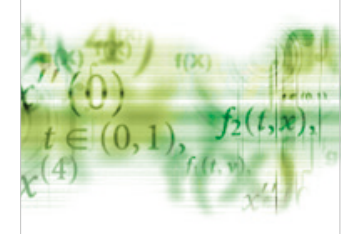

International Journal of

Differential Equations

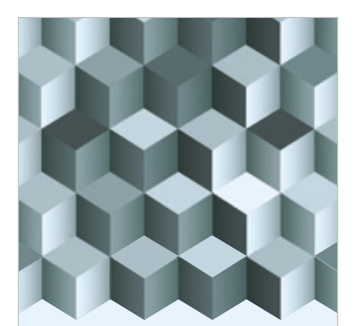

Journal of

Function Spaces
The Scientific

World Journal

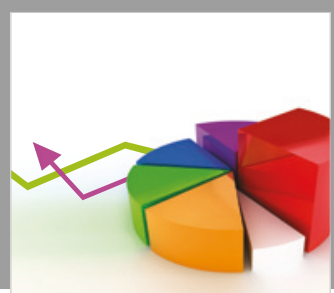

Journal of

Probability and Statistics
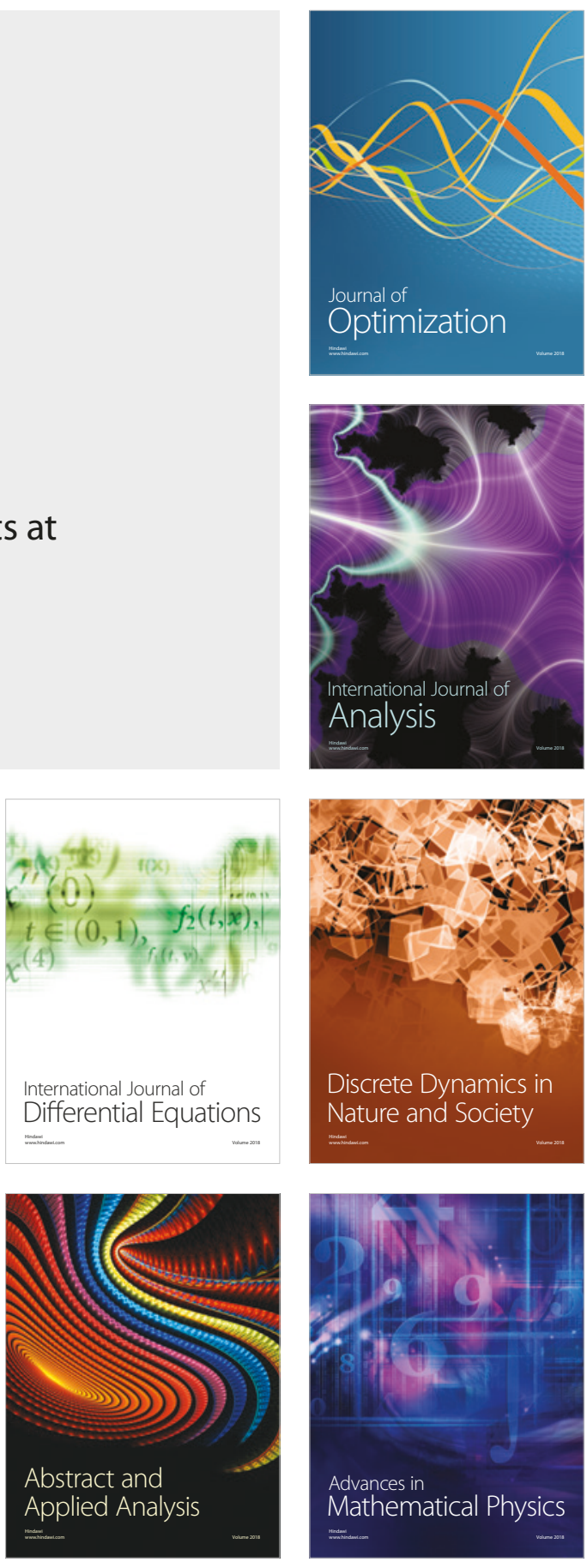